\title{
Dipeptidyl Peptidase-4 Inhibitor Switching as an Alternative Add-on Therapy to Current Strategies Recommended by Guidelines: Analysis of a Retrospective Cohort of Type 2 Diabetic Patients
}

Masami Tanaka*, Takeshi Nishimura, Risa Sekioka and Hiroshi Itoh

Department of Internal Medicine, School of Medicine, Keio University 35 Shinanomachi, Shinjuku-ku, Tokyo 160-8582, Japan

\begin{abstract}
Objective: This retrospective cohort study aimed to investigate the significance of dipeptidyl peptidase-4 (DPP4) inhibitor switch therapy, which is currently not recommended by major diabetes guidelines.

Methods: The subjects were 238 outpatients with type 2 diabetes who had been prescribed sitagliptin 50 mg daily, which was subsequently changed in one of three ways. Patients whose sitagliptin was switched to vildagliptin $50 \mathrm{mg}$ twice daily were defined as the switched group. Patients whose sitagliptin was increased to $100 \mathrm{mg}$ once daily were defined as the increased group. Patients who received an additional alfa-glucosidase inhibitor ( $\alpha-\mathrm{Gl}$ ) three times daily prior to meals and sitagliptin $50 \mathrm{mg}$ once daily were defined as the added group. The primary endpoint was the glycated hemoglobin $(\mathrm{HbA} 1 \mathrm{c})$ value at 6 months after the medication change. Patients whose oral hypoglycemic agents were changed within 6 months after switching to vildagliptin, increasing sitagliptin, or adding a-GI were excluded from the full analysis set and the remaining patients were included in the per protocol analysis.

Results: The per protocol analysis revealed that the $\mathrm{HbA1c}$ level decreased significantly in the switched group $(n=71)$ and the added group $(n=18)$ but did not change significantly in the increased group $(n=69)$. Analysis of the full set showed that the $\mathrm{HbA1c}$ level decreased significantly in all three groups (switched [n=92], increased [ $n=88$ ], and added $[n=25])$.

Conclusion: Switching DPP-4 inhibitors can adequately reduce HbA1c compared with increasing original DPP4 inhibitor dose or adding an $\alpha-G I$. When the original DPP-4 inhibitor did not significantly improve glycemic control, making a DPP-4 inhibitor switch, which is not recommended by major diabetes guidelines, can be an alternative strategy.
\end{abstract}

Keywords: Dipeptidyl peptidase-4; Oral hypoglycemic agent; Medication adherence; Sitagliptin; Vildagliptin; Safety; Medical cost

\section{Introduction}

Diabetes mellitus is a chronic and progressive disease that poses a growing public health problem worldwide. Patients who have diabetes long term require many classes of oral hypoglycemic agents (OHAs), often at relatively high doses, because glycemic control decreases with age [1].

Diabetes guidelines issued by American Diabetes Association (ADA) and European Association for the Study of Diabetes (EASD) recommend metformin as the first-line medication for type 2 diabetes mellitus [2]. However, it is usually difficult for diabetic patients to achieve glycemic control goals using metformin only. In such cases, $\mathrm{ADA}$ and EASD guidelines recommend add-on therapy, which is the addition of another class of hypoglycemic agents to metformin [2].

Dipeptidyl peptidase-4 (DPP-4) inhibitors have been used widely because of their high efficacy, low risk of hypoglycemia, and neutral effect on body weight $[3,4]$. In fact, DPP-4 inhibitors have generally been accepted as the second- or third-line therapies in patients receiving first-line metformin therapy [2]. However, a step-up strategy from regimens including DPP-4 inhibitors has not yet been established. In the clinical setting, glycemic control sometimes improves after the switch to a different DPP-4 inhibitor. Many doctors exchange DPP-4 inhibitors rather than add other classes of OHAs, which are expected to exert additive glycated hemoglobin (HbAlc)-lowering effects through mechanisms that differ from those of DPP-4 inhibitors. The reason for this is not clear. There are no theoretical bases for the efficacy of this practice, and the current guidelines do not provide clinical advice relating to DPP-4 inhibitor switching.

Alfa-glucosidase inhibitor ( $\alpha-G I)$, the use of which is not currently recommended by the ADA/EASD guidelines, is a readily available OHA whose usefulness is not limited by patient age or renal function in contrast to other OHAs available in Japan. Although its gastrointestinal adverse effects are common, this class of medicine is relatively safe and effective for improving postprandial hyperglycemia [5]. Additionally, this class of OHA is shown to reduce the risk of cardiovascular diseases [6].

Considering these circumstances, we focused on diabetic patients taking sitagliptin $50 \mathrm{mg}$ daily, the standard dose of this drug in Japan, who displayed inadequate glycemic control. The objectives of this study were to evaluate the effectiveness and safety of DPP-4 inhibitor

*Corresponding author: Masami Tanaka, Department of Internal Medicine, Schoo of Medicine, Keio University 35 Shinanomachi, Shinjuku-ku, Tokyo 160-8582, Japan Tel: 81-3-5363-3797; Fax: 81-3-3359-2745; E-mail: tana176k@keio.jp

Received August 26, 2016; Accepted September 06, 2016; Published September 12, 2016

Citation: Tanaka M, Nishimura T, Sekioka R, Itoh H (2016) Dipeptidyl Peptidase-4 Inhibitor Switching as an Alternative Add-on Therapy to Current Strategies Recommended by Guidelines: Analysis of a Retrospective Cohort of Type 2 Diabetic Patients. J Diabetes Metab 7: 701. doi: 10.4172/2155-6156.1000701

Copyright: $\odot 2016$ Tanaka M, et al. This is an open-access article distributed under the terms of the Creative Commons Attribution License, which permits unrestricted use, distribution, and reproduction in any medium, provided the original author and source are credited. 
Citation: Tanaka M, Nishimura T, Sekioka R, Itoh H (2016) Dipeptidyl Peptidase-4 Inhibitor Switching as an Alternative Add-on Therapy to Current Strategies Recommended by Guidelines: Analysis of a Retrospective Cohort of Type 2 Diabetic Patients. J Diabetes Metab 7: 701. doi: $10.4172 / 2155-6156.1000701$

Page 2 of 5

switch therapy, which is currently not recommended by diabetes guidelines, and validate the feasibility of this regimen. We compared three regimens: 1) switching sitagliptin to vildagliptin; 2) increasing sitagliptin dose; and 3) adding a-GI to sitagliptin. Specifically, we examined changes in $\mathrm{HbAlc}$, body weight, liver function, and kidney function.

\section{Patients and Methods}

\section{Study design}

This retrospective cohort study analyzed data from the medical records of 238 outpatients with type 2 diabetes mellitus who attended the Department of Internal Medicine, Keio University Hospital, between November 2011 and October 2014. The primary endpoint was the HbAlc value at 6 months after the medication change. The secondary endpoints were changes in body weight, aspartate aminotransferase (AST), alanine aminotransferase (ALT), and estimated glomerular filtration rate (eGFR) from baseline to 6 months after the medication change.

Inclusion criteria were: 1) patients prescribed sitagliptin $50 \mathrm{mg}$ daily (in the morning) for at least 2 months without any changes to their OHAs; and 2) medication was subsequently changed in one of the following three ways. Patients whose sitagliptin was switched to vildagliptin $100 \mathrm{mg}$ daily $(50 \mathrm{mg}$ in the morning and $50 \mathrm{mg}$ in the evening) were defined as the switched group. Patients whose sitagliptin was increased to $100 \mathrm{mg}$ daily (in the morning) were defined as the increased group. Patients who received an additional $\alpha$-GI (three times just before meals) with sitagliptin $50 \mathrm{mg}$ daily were defined as the added group regardless of $a$-GI type (acarbose, voglibose, or miglitol) or dose. The patients were excluded from the analysis if they exhibited poor drug compliance (assessed from medical record information), their $\mathrm{HbA} 1 \mathrm{c}$ value was $<6.5 \%$, their eGFR was $<30 \mathrm{~mL} / \mathrm{min} / 1.73 \mathrm{~m}^{2}$, AST or ALT were $>3$ times upper limit, they received cancer medication, they were referred to another hospital, or they were lost to follow-up. A flow chart of the patient enrollment process is shown in Figure 1.

The protocol was approved by the Ethics Committee of Keio University School of Medicine and conducted in accordance with the Declaration of Helsinki.

\section{Data collection}

Basic demographic data were collected for all patients from their medical records, including sex, age, height, weight, duration of diabetes, baseline HbA1c, eGFR, AST, ALT, and systolic and diastolic blood pressure. Body mass index (BMI) was calculated as weight $(\mathrm{kg})$ divided by height $(\mathrm{m})$ squared $\left(\mathrm{kg} / \mathrm{m}^{2}\right)$.

All measurements were performed by the Department of Laboratory Medicine of Keio University School of Medicine using routine automated laboratory methods as previously described [7]. The HbAlc level was expressed in accordance with the National Glycohemoglobin Standardization Program guidelines as recommended by the Japanese Diabetes Society [8]. Estimated glomerular filtration rate (eGFR)

\begin{tabular}{|l|}
\hline Switched Group \\
Screened $N=110$ \\
Switching from sitagliptin $50 \mathrm{mg}$ to vildagliptin $100 \mathrm{mg}$ \\
\hline
\end{tabular}

Increased Group

Screened $N=100$

Increasing sitagliptin doses from $50 \mathrm{mg}$ to $100 \mathrm{mg}$

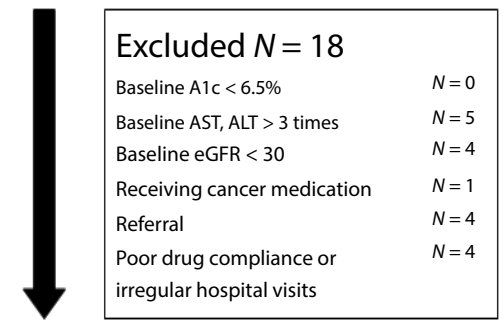

Full analysis set (FAS)
$N=92$

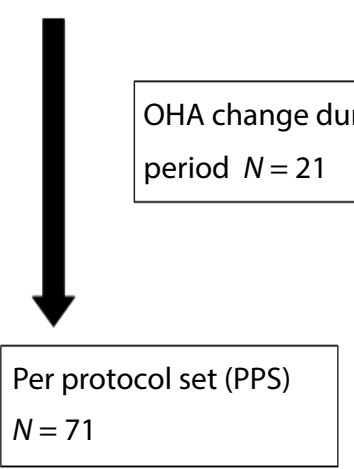

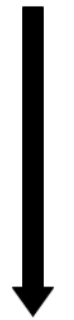

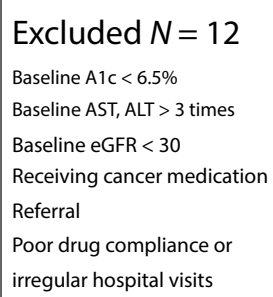

Full analysis set (FAS)

$N=88$

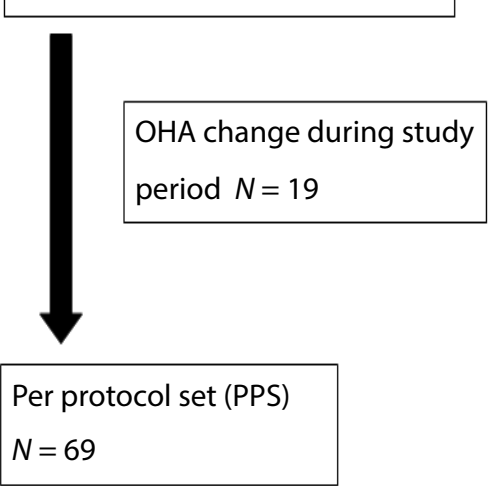

\section{Added Group \\ Screened $N=28$ \\ Adding $\alpha$-Gl to antidiabetic therapy of sitagliptin $50 \mathrm{mg}$}

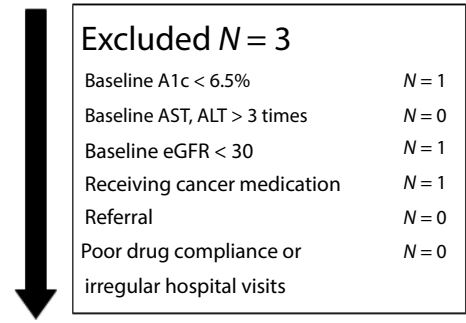

Full analysis set (FAS)

$N=25$

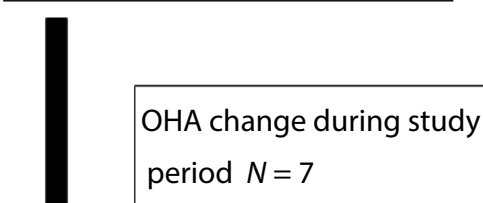

Per protocol set (PPS)

$N=18$

Figure 1: Flow chart of the patient selection process. AST: Aspartate Aminotransferase; ALT: Alanine Aminotransferase; eGFR: Estimated Glomerular Filtration Rate; OHA: Oral Hypoglycemic Agent. 
Citation: Tanaka M, Nishimura T, Sekioka R, Itoh H (2016) Dipeptidyl Peptidase-4 Inhibitor Switching as an Alternative Add-on Therapy to Current Strategies Recommended by Guidelines: Analysis of a Retrospective Cohort of Type 2 Diabetic Patients. J Diabetes Metab 7: 701 . doi: $10.4172 / 2155-6156.1000701$

Page 3 of 5

$\left(\mathrm{mL} / \mathrm{min} / 1.73 \mathrm{~m}^{2}\right)$ was calculated according to the Statement of the Japan Nephrology Society [9] as follows: eGFR ( $\mathrm{mL} / \mathrm{min} / 1.73$ $\left.\mathrm{m}^{2}\right)=194 \times(\text { serum creatinine })^{-1.094} \times(\text { age })^{-0.287}(\times 0.739$ for women $)$

\section{Statistical methods}

As shown in Figure 1, 92 patients of the switched group, 88 of the increased group, and 25 of the added group were included in the full analysis set. Patients whose OHAs were changed within 6 months after switching to vildagliptin, increasing sitagliptin to $100 \mathrm{mg}$, or adding a-GI were excluded from the full analysis set, while the remaining patients were included in the per protocol set analyses. The primary endpoint was evaluated using both sets, while the secondary endpoints were evaluated using the per protocol sets only. Changes in HbAlc were investigated using repeated-measures analysis of variance. The comparison of body weights as well as AST, ALT, and eGFR levels before and after the study period were analyzed using paired t-tests. These analyses were performed using SPSS (version 21.0; SPSS Inc., Chicago, IL, USA). Data are expressed as mean \pm standard deviation (SD) in the text and tables. Values of $\mathrm{P}<0.05$ were considered statistically significant.

\section{Results}

\section{Patient characteristics}

Patient demographic and laboratory data are shown in Table 1 . The average ages of patients in the switched, increased, and added groups were $68.5,67.0$, and 71.8 years, respectively, indicating that many old patients were included in this study.

\section{Changes in $\mathrm{HbAlc}$ in each group}

The changes in HbAlc are shown in Table 2. The per protocol analysis revealed that the $\mathrm{HbAlc}$ level decreased significantly in the switched and added groups but did not change significantly in the increased group. The full analysis set showed that the HbAlc level decreased significantly in all three groups.

\section{Safety parameters by group}

The time courses of clinical parameters are presented in Table 3. Body weight significantly decreased in the increased group $(\mathrm{P}<0.05)$. None of the other clinical parameters changed significantly.

\section{Discussion}

The present study compared the effects among vildagliptin 100 $\mathrm{mg}$ daily (50 $\mathrm{mg}$ in the morning and $50 \mathrm{mg}$ in the evening), sitagliptin $100 \mathrm{mg}$ daily (once in the morning), or combination of sitagliptin 50 $\mathrm{mg}$ and $\alpha$-GI (three times just before meals) on glycemic control in patients with type 2 diabetes who did not achieve adequate glycemic control with sitagliptin $50 \mathrm{mg}$ daily (once in the morning) alone. The per protocol analyses showed that switching to vildagliptin $100 \mathrm{mg}$ daily and adding $\alpha$-GI to sitagliptin $50 \mathrm{mg}$ daily significantly lowered $\mathrm{HbA1c}$ levels. On the other hand, increasing $50 \mathrm{mg}$ sitagliptin to 100 mg daily did not improve HbA1c levels. The full set analysis showed that all three regimens decreased $\mathrm{HbA1c}$ levels.

The per protocol analysis results are consistent with those of several previous reports. It was reported that sitagliptin $50 \mathrm{mg}$ daily and 100 mg daily showed similar improvements in HbAlc [10]. On the other hand, a possible superiority of vildagliptin over other DPP-4 inhibitors regarding a hypoglycemic effect $[11,12]$ was also reported. The $\mathrm{IC}_{50}$ for vildagliptin and sitagliptin, the concentration required to achieve
$50 \%$ inhibition of DPP-4 activity, is reportedly $5 \mathrm{nmol} / \mathrm{L}$ [13] and 26.3 $\mathrm{nmol} / \mathrm{L}[14]$, respectively, which suggests that vildagliptin might be a potent DPP-4 inhibitor. Accordingly, a previous study showed that elevated levels of intact incretin hormones are maintained for a longer period following the administration of vildagliptin than that following the administration of sitagliptin [15]. Nonetheless, the evidence of superiority of one medicine over another can be only derived from head-to-head prospective trials.

The addition of $\alpha$-GI to a DPP- 4 inhibitor is an effective diabetes treatment strategy that leads to a $0.3-0.5 \% \mathrm{HbAlc}$ improvement [1618]. Alfa-GI increases glucagon-like peptide-1 secretion from $L$ cells in the lower duodenum, while DPP-4 inhibitor inhibits glucagon-like peptide-1 degradation, potentiating its hypoglycemic activity, meaning that this combination therapy is quite effective.

The results of this study do not mean that a changeover from sitagliptin to vildagliptin is effective for all patients with type 2 diabetes; rather, only those patients who do not respond adequately to sitagliptin might benefit from this transition. The pharmacokinetics/ pharmacodynamics of sitagliptin and vildagliptin differ among patients. If the patients respond well to sitagliptin, it is difficult to obtain an increased effect by switching to vildagliptin because the DPP4 inhibition rate already peaked with sitagliptin use in such patients.

The prices of the drugs appeared in this paper at present in Japan (since April 2016) are as follows. Sitagliptin $50 \mathrm{mg}$ tablet, 136.50 yen; sitagliptin $100 \mathrm{mg}$ tablet, 205.40 yen; vildagliptin $50 \mathrm{mg}$ tablet, 80.10 yen; acarbose $50 \mathrm{mg}$ tablet, 20.20 yen; miglitol $5 \mathrm{mg}$ tablet, 52.40 yen; and voglibose $0.2 \mathrm{mg}$ tablet, 35.00 yen. Therefore, vildagliptin $100 \mathrm{mg}$ ( 2 of $50 \mathrm{mg}$ tablet, $80.10 \times 2=160.20$ yen) is cheaper than sitagliptin $100 \mathrm{mg}$ (205.40 yen) or sitagliptin $50 \mathrm{mg}$ plus $\alpha$-GI three times daily (acarbose $136.50+20.20 \times 3=197.10$ yen, miglitol $136.50+52.40 \times 3=293.7$ yen, voglibose $136.50+35.00 \times 3=241.50$ yen). Accordingly, the switched group featured the most favorable medical cost.

Patients in the switched group took vildagliptin twice daily. Patients in the added group took $\alpha$-GI three times daily in addition to sitagliptin

\begin{tabular}{|c|c|c|c|}
\hline & Switched & Increased & Added \\
\hline $\mathrm{N}$ & 92 & 88 & 25 \\
\hline Male sex, $\mathrm{n}(\%)$ & $56(60.9)$ & $59(67.0)$ & $14(56.0)$ \\
\hline Age (years) & $68.5 \pm 10.5$ & $67.0 \pm 11.0$ & $71.8 \pm 8.6$ \\
\hline Diabetes duration (years) & $17.5 \pm 9.2$ & $16.5 \pm 6.9$ & $16.9 \pm 9.5$ \\
\hline BMI (kg/m $\left.{ }^{2}\right)$ & $24.9 \pm 4.7$ & $25.6 \pm 4.1$ & $24.3 \pm 4.2$ \\
\hline SBP $(\mathrm{mmHg})$ & $133 \pm 15$ & $134 \pm 18$ & $132 \pm 16$ \\
\hline DBP $(\mathrm{mmHg})$ & $76 \pm 12$ & $76 \pm 11$ & $74 \pm 9$ \\
\hline
\end{tabular}

Table 1: Patients' baseline characteristics by study group values are expressed as number $(n)(\%)$ or mean \pm SD. Switched: Switched group; Increased: Increased group; Added: Added group; BMI: Body Mass Index; SBP: Systolic Blood Pressure; DBP: Diastolic Blood Pressure.

\begin{tabular}{|c|c|c|c|c|}
\hline \multirow{3}{*}{ PPS } & & Before & $\mathbf{6}$ months & P value \\
\hline \multirow{3}{*}{ FAS } & Switched & $8.15 \pm 0.83$ & $7.86 \pm 0.86$ & $<0.001$ \\
\cline { 2 - 5 } & Increased & $8.13 \pm 0.80$ & $7.95 \pm 1.04$ & 0.119 \\
\cline { 2 - 5 } & Added & $8.26 \pm 0.88$ & $7.66 \pm 0.99$ & $<0.001$ \\
\cline { 2 - 5 } & Switched & $8.22 \pm 0.88$ & $7.88 \pm 0.86$ & $<0.001$ \\
\cline { 2 - 5 } & Increased & $8.16 \pm 0.81$ & $7.88 \pm 0.97$ & 0.014 \\
\cline { 2 - 5 } & Added & $8.18 \pm 0.92$ & $7.53 \pm 0.90$ & $<0.001$ \\
\hline
\end{tabular}

Table 2: Mean HbA1c level by study group. Values are expressed as mean $\pm \mathrm{SD}$ HbA1c, Glycated Hemoglobin; Increased: Increased group; Switched: Switched group; Added: Added group; Before, value at baseline; 6 months, value at 6 months after medication change; PPS: Per Protocol Set; FAS: Full Analysis Set. 
Citation: Tanaka M, Nishimura T, Sekioka R, Itoh H (2016) Dipeptidyl Peptidase-4 Inhibitor Switching as an Alternative Add-on Therapy to Current Strategies Recommended by Guidelines: Analysis of a Retrospective Cohort of Type 2 Diabetic Patients. J Diabetes Metab 7: 701. doi: 10.4172/2155-6156.1000701

Page 4 of 5

\begin{tabular}{|c|c|c|c|c|c|c|c|c|c|}
\hline & \multicolumn{3}{|c|}{ Switched } & \multicolumn{3}{|c|}{ Increased } & \multicolumn{1}{|c|}{ Added } \\
\hline & Before & After & $P$ & Before & After & $P$ & Before & After & $P$ \\
\hline BW & $67.0 \pm 15.4$ & $67.3 \pm 15.2$ & 0.066 & $68.0 \pm 12.9$ & $67.0 \pm 12.5$ & 0.037 & $62.3 \pm 9.8$ & $61.6 \pm 10.2$ & 0.091 \\
\hline AST (IU/L) & $25.9 \pm 12.2$ & $26.3 \pm 12.0$ & 0.682 & $26.8 \pm 13.2$ & $26.9 \pm 11.2$ & 0.915 & $29.3 \pm 20.3$ & $25.5 \pm 10.1$ & 0.175 \\
\hline ALT (IU/L) & $26.7 \pm 17.0$ & $26.5 \pm 18.0$ & 0.929 & $28.4 \pm 16.1$ & $29.5 \pm 17.9$ & 0.405 & $31.0 \pm 25.7$ & $29.5 \pm 18.6$ & 0.572 \\
\hline $\begin{array}{c}\text { eGFR (mL/ } \\
\left.\text { min/1.73m }{ }^{2}\right)\end{array}$ & $67.9 \pm 18.9$ & $66.5 \pm 17.5$ & 0.137 & $69.4 \pm 17.9$ & $68.7 \pm 16.4$ & 0.364 & $69.8 \pm 15.7$ & $68.7 \pm 16.6$ & 0.458 \\
\hline
\end{tabular}

Table 3: Changes in clinical parameters by study group. Values are expressed as mean \pm SD. Switched: Switched group; Increased: Increased group; Added; Added group; before: Baseline value; after: Value at the end of the study period; BW: Body Weight; AST: Aspartate Aminotransferase; ALT: Alanine Aminotransferase.

once daily. Therefore, the patients in the switched group had a simpler regimen, which means a lower medication frequency and fewer tablets, than those in the added group. Treatment adherence generally deteriorates as frequency and number of daily doses increases $[19,20]$; thus, simplifying dosing regimens improves medication adherence [21]. Furthermore, patients taking a-GI sometimes complain of gastrointestinal symptoms such as constipation or diarrhea. Although mild in most cases, such side effects can deteriorate patient quality of life. A switch from a DPP-4 inhibitor to vildagliptin might be more favorable than the addition of $\alpha-\mathrm{GI}$ in such cases.

Many older patients were included in this study. The number of old and fragile diabetic patients is increasing worldwide. Such patients often suffer from vital organ insufficiencies and tend to be vulnerable to the adverse events of diabetic medications. For such patients, monotherapy with a DPP-4 inhibitor is relatively safe and valuable compared to combination therapy since fewer potential adverse events are involved.

Among the secondary endpoints of this study, the only significant change was a decrease in body weight in the increased group. Although statistically significant, this change was minimal and appeared not to be clinically relevant. AST, ALT, and eGFR did not change significantly. None of the regimens worsened liver or kidney function. We observed no drug-related adverse events that led to drug discontinuation.

There are several limitations to the present study. First, because of its retrospective design and limited number of patients, the existence of confounding factors and biases cannot be ruled out. Second, even though patients who switched from sitagliptin to vildagliptin showed a significantly decreased HbAlc level, we cannot draw any conclusions about the superiority/inferiority about these DPP-4 inhibitors as mentioned above. Finally, the participants of this study were patients who attend a university hospital, who are special in some regard. Therefore, the study's results may not be applicable to the general population or to diabetic patients who are treated in primary care settings.

In conclusion, switching DPP-4 inhibitors adequately lowers $\mathrm{HbA1c}$ levels compared with increasing the dose of the original DPP-4 inhibitor or adding another OHA class. By switching DPP-4 inhibitors, advantages including decreased medical cost, increased patient adherence, and increased safety can be anticipated. When the firstline DPP-4 inhibitor does not adequately improve glycemic control, a DPP-4 inhibitor switch, which is not currently recommended by major diabetes guidelines, can be an alternative strategy.

\section{References}

1. U.K. Prospective Diabetes Study Group U.K. (1995) Prospective Diabetes Study 16: Overview of 6 years' therapy of type II diabetes: a progressive disease. Diabetes 44: 1249-1258.

2. Inzucchi SE, Bergenstal RM, Buse JB, Diamant M, Ferrannini E, et al. (2015) Management of hyperglycemia in type 2 diabetes, 2015: a patient-centered approach: update to a position statement of the American Diabetes Association and the European Association for the Study of Diabetes. Diabetes Care 38 140-149.

3. Barnett A (2006) DPP-4 inhibitors and their potential role in the management of type 2 diabetes. Int J Clin Pract 60: 1454-1470.

4. Amori RE, Lau J, Pittas AG (2007) Efficacy and safety of incretin therapy in type 2 diabetes: systematic review and meta-analysis. JAMA 298: 194-206.

5. Chiasson JL, Josse RG, Hunt JA, Palmason C, Rodger NW, et al. (1994) The efficacy of acarbose in the treatment of patients with non-insulin-dependent diabetes mellitus. A multicenter controlled clinical trial. Ann Intern Med 121 928-935.

6. Chiasson JL, Josse RG, Gomis R, Hanefeld M, Karasik A, et al. (2003) Acarbose treatment and the risk of cardiovascular disease and hypertension in patients with impaired glucose tolerance: the STOP-NIDDM trial. JAMA 290: 486-494.

7. Tanaka M, Sekioka R, Nishimura T, Kawai T, Meguro S, et al. (2015) Serum $\mathrm{C}$-peptide to plasma glucose ratio may be associated with efficacy of vildagliptin in Japanese patients with type 2 diabetes mellitus. Diabetology International 6: 197-205.

8. Committee of the Japan Diabetes Society on the Diagnostic Criteria of Diabetes Mellitus; Seino Y, Nanjo K, Tajima N, Kadowaki T, et al. (2010) Report of the committee on the classification and diagnostic criteria of diabetes mellitus. J Diabetes Invest 1: 212-228.

9. Matsuo S, Imai E, Horio M, Yasuda Y, Tomita K, et al. (2009) Collaborators developing the Japanese equation for estimated GFR. Revised equations for estimated GFR from serum creatinine in Japan. Am J Kidney Dis 53: 982-992.

10. Iwamoto Y, Taniguchi T, Nonaka K, Okamoto T, Okuyama K, et al. (2010) Dose-ranging efficacy of sitagliptin, a dipeptidase-4 inhibitor, in Japanese patients with type 2 diabetes mellitus. Endocrine J 57: 383-394.

11. Signorovitch JE, Wu EQ, Swallow E, Kantor E, Fan L, et al. (2011) Comparative efficacy of vildagliptin and sitagliptin in Japanese patients with type 2 diabetes mellitus. A matching-adjusted indirect comparison of randomized trials. Clin Drug Invest 31: 665-674.

12. Aroda VR, Henry RR, Han J, Huang W, DeYoung MB, et al. (2012) Efficacy of GLP-1 receptor agonists and DPP-4 inhibitors: meta-analysis and systematic review. Clin Ther 34: 1247-1258.

13. He YL, Yamaguchi M, Ito H, Terao S, Sekiguchi K (2010) Pharmacokinetics and pharmacodynamics of vildagliptin in Japanese patients wiith Type 2 diabetes. Int J Clin Pharmacol Ther 48: 582-595

14. Bergman AJ, Stevens C, Zhou Y, Yi B, Laethem M, et al. (2006) Pharmacokinetic and pharmacodynamics properties of multiple oral doses of sitagliptin, a dipeptidyl peptidase-IV inhibitor: a double-blind, randomized placebo-controlled study in healthy male volunteers. Clin Ther 28: 55-72.

15. Marfella R, Barbieri M, Grella R, Rizzo MR, Nicoletti GF, et al. (2010) Effects of vildagliptin twice daily vs. sitagliptin once daily on 24-hour acute glucose fluctuations. J Diabetes Complicat 24: 79-83.

16. Iwamoto Y, Kashiwagi A, Yamada N, Terao S, Mimori N, et al. (2010) Efficacy and safety of vildagliptin and voglibose in Japanese patients with type 2 diabetes: a 12-week, randomized, double-blind, active-controlled study. Diabetes Obes Metab 12: 700-708.

17. Vichayanrat A, Ploybutr S, Tunlakit M, Watanakejorn P (2002) Efficacy and safety of voglibose in comparison with acarbose in type 2 diabetic patients. Diabetes Res Clin Pract 55: 99-103.

18. Johnston PS, Lebovitz HE, Coniff RF, Simonson DC, Raskin P, et al. (1998) Advantages of alpha-glucosidase inhibition as monotherapy in elderly type 2 diabetic patients. J Clin Endocrinol Metab 83: 1515-1522. 
Citation: Tanaka M, Nishimura T, Sekioka R, Itoh H (2016) Dipeptidyl Peptidase-4 Inhibitor Switching as an Alternative Add-on Therapy to Current Strategies Recommended by Guidelines: Analysis of a Retrospective Cohort of Type 2 Diabetic Patients. J Diabetes Metab 7: 701. doi: 10.4172/2155-6156.1000701

Page 5 of 5

19. Claxton AJ, Cramer J, Pierce C (2001) A systematic review of the associations between dose regimens and medication compliance. Clin Ther 23: 1296-1310.

20. Paes AH, Bakker A, Soe-Agnie CJ (1997) Impact of dosage frequency on patient compliance. Diabetes Care 20: 1512-1517.
21. Schroeder K, Fahey T, Ebrahim S (2004) How can we improve adherence to blood pressure-lowering medication in ambulatory care? Arch Intern Med 164: 722-732. 